\title{
Entrer en collection. Pour une ethnographie des gestes et des techniques de collecte
}

Entering a collection. Towards an ethnography of the gestures and techniques of collecting

\section{Julien Bondaz}

\section{CpenEdition} Journals

Édition électronique

URL : http://journals.openedition.org/cel/481

DOI : $10.4000 /$ cel.481

ISSN : 2262-208X

Éditeur

École du Louvre

\section{Édition imprimée}

Date de publication : 1 avril 2014

Référence électronique

Julien Bondaz, «Entrer en collection. Pour une ethnographie des gestes et des techniques de collecte », Les Cahiers de l'École du Louvre [En ligne], 4 | 2014, mis en ligne le 01 avril 2014, consulté le 11 octobre 2018. URL : http://journals.openedition.org/cel/481 ; DOI : 10.4000/cel.481

\section{c) (i) () $\Theta$}

Les Cahiers de l'École du Louvre sont mis à disposition selon les termes de la licence Creative Commons Attribution - Pas d'Utilisation Commerciale - Pas de Modification 4.0 International. 


\section{Cahiers de l'École du Louvre}

recherches en histoire de l'art, histoire des civilisations archéologie, anthropologie et muséologie

Numéro 4. Avril 2014

\section{Entrer en collection.}

Pour une ethnographie des gestes

et des techniques de collecte

Julien Bondaz

Article disponible en ligne à l'adresse :

http://www.ecoledulouvre/cahiers-de-l'ecole-du-louvre/numero4avril2014/Bondaz.pdf

Pour citer cet article :

Julien Bondaz, «Entrer en collection. Pour une ethnographie des gestes et des techniques de collecte », Cahiers de l'Ecole du Louvre, recherches en histoire de I'art, histoire des civilisations, archéologie, anthropologie et muséologie [en ligne] $n^{\circ} 4$, avril 2014, p. 24 à 32.

\section{cc) creative \\ B.: $\Theta \$$}

(C) École du Louvre

Cet article est mis à disposition selon les termes de la Licence Creative Commons Attribution - Pas d'utilisation commerciale - Pas de modification 3.0 non transposé. 


\section{Cahiers de l'École du Louvre recherches en histoire de l'art, histoire des civilisations archéologie, anthropologie et muséologie \\ Numéro 4. Avril 2014}

Sommaire

Éditorial

p. 1

\section{Étude}

Le musée de Sculpture comparée au prisme de la collection de cartes postales éditées par les frères Neurdein (1904-1915)

Dominique Jarrassé et Emmanuelle Polack

p. $2-20$

Dossier:

Les modalités de la collecte : rapt, troc, marché, fouilles, don...

Et leur impact sur l'objet

Dans collection, il y a collecte...

Introduction de Dominique Jarrassé.

p. $21-23$

Entrer en collection. Pour une ethnographie des gestes et des techniques de collecte

Julien Bondaz

p. $24-32$

De la meilleure façon de constituer une collection. Le cas des émaux «byzantins » de Mikhaill Botkine

Aglaé Achechova.

Edme Antoine Durand (1768-1835) : un bâtisseur de collections

Louise Detrez p. $45-55$

Réunir une documentation pour l'Art Brut : les prospections de Dubuffet dans l'immédiat après-guerre au regard du modèle ethnographique Baptiste Brun p. $56-66$

Travailler sur des objets en faisant se confronter les points de vue : regards croisés sur le langage fou : l'exemple des écrits bruts

Vincent Capt p. $67-75$

D’un regard-pilote à l'écart. L'impact de Dubuffet sur les collectes de l'association L'Aracine

Déborah Couette. p. $76-86$

La cabane éclatée. Morcellement des objets immobiliers apparentés à l'art brut

Roberta Trapani .p. $87-95$ 


\section{Entrer en collection. \\ Pour une ethnographie des gestes \\ et des techniques de collecte}

\section{Julien Bondaz}

Une définition pragmatique de l'idée de collecte pourrait être formulée ainsi : la collecte désigne la transformation d'objets - artefacts ou produits de la nature - en objets de collection. Entendue ainsi, elle constitue un moment de leur biographie sociale ou culturelle ${ }^{1}$ ou une étape de leur carrière ${ }^{2}$, et génère un ensemble de requalifications, de modifications physiques, de réemplois, de nouveaux usages, de changements de valeurs, et plus largement de transferts culturels et conceptuels. Une telle définition de l'idée de collecte oblige par ailleurs à penser les liens étroits qui existent entre les modes d'acquisition des objets et les pratiques de collection. Une première difficulté surgit alors, qui concerne les figures du collecteur et du collectionneur, dans la mesure où elles peuvent selon les cas désigner soit une seule et même personne, soit deux personnes distinctes, soit encore une personne et une institution ${ }^{3}$. Or, si les travaux d'historiens, d'anthropologues ou d'ethnologues consacrés aux pratiques de collection sont nombreux, la question des techniques de collecte a été jusqu'à présent beaucoup moins étudiée. À partir des années 1970, dans le cadre du tournant réflexif en anthropologie, plusieurs études ont été consacrées aux pratiques de terrain des ethnologues et aux fondements ou aux développements de l'ethnographie muséale, initiant ainsi des réflexions stimulantes concernant l'histoire des collectes ethnographiques et mettant l'accent sur les grandes missions ethnographiques c'est le cas de la fameuse Mission Dakar-Djibouti conduite sous la direction de Marcel Griaule entre 1931 et $1933^{4}$. Des historiens des sciences ont insisté sur l'idée de "collecte du monde " qui animait les explorateurs entre le $\mathrm{xvI}^{\mathrm{e}}$ et le $\mathrm{XIX}^{\mathrm{e}}$ siècle $^{5}$ ou se sont plus spécialement intéressés aux pratiques de collecte et de collection naturalistes ${ }^{6}$.

Depuis, l'influence des postcolonial studies a cependant introduit deux biais dans l'intérêt porté aux activités de collecte, conduisant souvent les chercheurs à focaliser leur attention sur les collectes se déroulant en situation coloniale et - ce qui se justifie moins mais s'explique en partie par ce premier biais - à amalgamer la grande variété des modes d'acquisition des objets en une pratique généralisée de pillage, qui donnerait ainsi à voir le pouvoir hégémonique des collecteurs, les ethnologues et les missionnaires étant les premiers visés par les critiques ${ }^{7}$. Il ne s'agit évidemment pas, comme nous le verrons plus loin, de nier les conditions parfois brutales des collectes coloniales, ni leur contexte politique, mais d'inviter à mettre en perspective les pratiques de collecte sur le temps long, en posant par exemple la question des continuités ou des ruptures provoquées par les

1. Arjun Appadurai (dir.), The social life of Things. Commodities in cultural perspectives, Cambridge, Cambridge University Press, 1986.

2. Christian Bromberger et Denis Chevallier (dir.), Carrières d'objets, Paris, Éditions de la MSH, 1999.

3. Il n'est pas inintéressant de noter qu'en anglais, la distinction entre la figure du collecteur et celle du collectionneur est rendue d'autant plus complexe qu'un même terme, collector, désigne les deux.

4. Voir par exemple Jean Jamin, « Objets trouvés des paradis perdus. À propos de la mission Dakar-Djibouti », dans Jacques Hainard et Roland Kaehr (éd.) Collections passion, Neuchâtel, musée d'Ethnographie, 1982, pp. 69-100.

5. Daniel Defert, «La collecte du monde : une étude des récits de voyages du XVI ${ }^{e}$ au XVII ${ }^{e}$ siècle », dans Jacques Hainard et Roland Kaehr (dir.), op. cit. note 4, pp. 17-31; Marie-Noëlle Bourguet, «La collecte du monde : voyage et histoire naturelle (fin XVII Blanckaert, Claudine Cohen, Pietro Corsi et Jean-Louis Fisher (coord.), Le Muséum au premier siècle de son histoire, Paris, Editions du Museum national d'histoire naturelle, 1997, pp. 163-196. 6. Bertrand Daugeron, Collections naturalistes entre science et empire (1763-1804), Paris, publications scientifiques du MNHN, 2009.

7. Depuis les années 1990, plusieurs chercheurs ont proposé des approches plus fines et plus stimulantes des collectes. Voir en particulier Nicholas Thomas, Entangled Objects. Exchange, Material Culture and Colonialism in Pacific, Cambridge, Mass., Harvard University Press, 1991 et Enid Schildkrout et Curtis A. Keim (eds.), The scramble for art in Central Africa, Cambridge University Press, 1998. 
décolonisations, et surtout à détailler les différentes modalités de collecte, c'està-dire la diversité des gestes et des techniques qui visent à faire entrer des objets en collection ${ }^{8}$. Envisager la collecte non pas comme un acte répété selon des modalités invariables ou dans un but unique, mais comme un faisceau d'actions et d'intentions complexes, permet notamment de se demander si la diversité des manières de transformer des artefacts ou des produits de la nature en objets de collection produit des variations dans les façons de voir, de qualifier et de valoriser ces objets. Autrement dit, il s'agit plus précisément d'esquisser des liens de causalité entre les gestes ou les techniques de collecte et les changements de statut et de valeur que subissent les objets collectés.

Ce programme relève en grande partie du défi, pour deux raisons principales. D'une part, la collecte, telle que nous l'avons définie, constitue moins un acte qu'un processus mettant en jeu des aspects variés, aussi bien techniques, cognitifs et affectifs, qu'administratifs ou légaux'. Faire l'ethnographie des pratiques de collecte apparait en ce sens comme le meilleur moyen de saisir ensemble ces différents aspects, en recourant par exemple aux notions de chaîne opératoire, empruntée à l'anthropologie des techniques, et de chaîne de médiations, dans le sens que lui ont donné la sociologie de la traduction et la sociologie pragmatique, ou encore aux outils d'analyse de la micro-histoire. D'autre part, la description des pratiques de collecte et de la complexité des processus en jeu qui vient d'être décrite est rendue d'autant plus difficile que les documents font généralement défaut et que les données sont lacunaires.

L'idéal reste évidemment d'ethnographier des missions de collecte contemporaines, en observant les pratiques des collecteurs et en analysant leurs discours. Les ethnologues qui participent à de telles missions sont heureusement nombreux à porter un regard réflexif sur leurs propres pratiques et à expliciter les procédures d'acquisition auxquelles ils ont recours, par un souci éthique aujourd'hui essentiel ${ }^{10}$. Mais, lorsqu'il s'agit de documenter des pratiques de collecte anciennes, les archives constituent le terrain principal de l'historien ou de l'ethnologue et les sources font souvent défaut. Le propos de cet article n'est cependant pas là : l'objectif est de proposer quelques pistes de réflexion générale sur la manière dont les variétés que l'on observe dans les techniques et les gestes de collecte produisent des différences dans les manières de voir et de comprendre les objets collectés. Les exemples historiques et ethnographiques mobilisés sont issus de recherches menées sur les pratiques de collecte et de collection en Afrique de l'Ouest. Faire l'ethnographie des gestes et des techniques de collecte permet de mieux comprendre comment des artefacts ou des produits de la nature sont requalifiés en pièces de collection, et plus particulièrement en objets ethnographiques ou en œuvres d'art.

\section{Suivre les instructions: le cadre normatif des collectes}

La période de disciplinarisation de l'ethnologie et de professionnalisation des ethnologues ${ }^{11}$, qui s'ouvre dans la première moitié du $\mathrm{XX}^{\mathrm{e}}$ siècle, est particulièrement intéressante du point de vue de l'histoire des collectes, non seulement parce qu'elle est marquée par l'organisation de nombreuses missions ethnographiques ayant entre autres objectifs de rassembler des collections pour

8. Voir par exemple Serge Reubi, «Pratiques de collection », dans Marc-Olivier Gonseth, Bernard Knodel et Serge Reubi (dir.), Retour d'Angola, Neuchâtel, Musée d'ethnographie, 2010, pp. 162178.

9. Christopher B. Steiner, "Rights of Passage. On the Liminal Identity of Art in the Border Zone", dans Fred R. Myers, The Empire of Things. Regimes of Value and Material Culture, Santa Fe, School of American Research Press, 2001, pp. 207-231.

10. Voir en particulier Anne-Marie Bouttiaux, «Récolter les "choses" et laisser les esprits en paix chez les Guro (Côte d'Ivoire) », A.-M. Bouttiaux (textes réunis par), Afrique : musées et patrimoines pour quels publics ?, Tervuren-Paris, Musée royal de I'Afrique centrale-KarthalaCulture Lab Editions, 2007, pp. 55-63. Ces ethnologues collecteurs apparaissent ainsi comme des figures de « passeurs de mémoire ». Voir à ce sujet Gaetano Ciarcia (dir.), Ethnologues et passéurs de mémoire, Paris-Montpellier, Karthala-MSHM, 2011.

11. Éric Jolly, « Marcel Griaule, ethnologue : la construction d'une discipline (1925-1956) 》, Journal des africanistes, 71(1), 2001, pp. 149-190 ; Emmanuelle Sibeud, « Ethnographie, ethnologie et africanisme. La "disciplinarisation" de l'ethnologie française dans le premier tiers du XXe siècle » dans J. Boutier, J.-C. Passeron et J. Revel (éds), Qu'est-ce qu'une discipline ?, Paris, Éditions de I'EHESS, 2006, pp. 229-245. 
les musées occidentaux, mais aussi parce qu'une série d'instructions ou de conseils sont rédigés à destination des collecteurs. Un lien historique étroit existe entre la mise en place de méthodes d'enquête spécifiques à l'ethnographie et la volonté d'organiser scientifiquement les collectes d'objets, qui permet ainsi de repérer un paradigme de la collecte dans l'institution de l'ethnologie ${ }^{12}$. En ce sens, les objets deviennent ethnographiques parce qu'ils sont collectés d'une certaine manière par les ethnographes ${ }^{13}$.

Le rôle de Marcel Mauss est ici central : les principes méthodologiques qu'il propose dans les années 1920, compilés plus tard dans un Manuel d'ethnographie publié de façon posthume ${ }^{14}$, sont particulièrement bien diffusés auprès de la première génération d'ethnologues professionnels français, qui sont presque tous ses étudiants. L'accent est alors mis sur l'importance de constituer des séries d'objets et de privilégier ceux qui sont les plus ordinaires ou les plus banals. C'est la fameuse " règle de la boîte de conserve ", selon laquelle " une boîte de conserve, par exemple, caractérise mieux nos sociétés que le bijou le plus somptueux ou que le timbre le plus rare ${ }^{15}$. Du point de vue des ethnographes, il s'agit pour le collecteur de lutter contre ses propres critères de jugements de goût dans la sélection des objets. Dans les Conseils aux chercheurs diffusés par l'Institut Français d'Afrique Noire après la Seconde Guerre mondiale, il est ainsi écrit : "Une collection ethnographique n'est pas une collection d'œuvres d'art rarissimes. Elle doit, bien au contraire, comprendre avant tout des objets appartenant véritablement à la vie réelle ${ }^{16}$. "Pendant la guerre, dans ses cours, Griaule insiste sur cette nécessité de suspendre son jugement de goût personnel :

"Encore une fois je pense qu'il est inutile de vous rappeler que vous devez faire abstraction de ce que vous vous trouvez beau. C'est tout de même la moindre des choses. [...] C'est l'un des domaines où l'ethnographe doit se dégager le plus de ses notions personnelles, c'est-à-dire des notions du groupe où il vit habituellement. [...]Pour constituer une collection je vous engage à recueillir les objets en ne partant pas de notre notion de beau mais en partant de la notion de beau de la société que vous étudiez. Il faut bien faire attention à cela, je ne saurai trop vous le répéter. Cela paraît très naturel quand vous écoutez votre cours, mais une fois qu'on est sur le terrain, on a tendance à se laisser aller à ses goûts, à dire : je ne veux pas rapporter ça, c'est trop moche, on se moquera de moi, etc. Vous avez tort. Pour ces gens, c'est peut-être beau. ${ }^{17}$

Des écarts entre ces instructions ou ces principes et les pratiques sur le terrain ne cessent cependant d'être observés, y compris à l'occasion des missions Griaule. Ils témoignent de l'importance des critères esthétiques dans les pratiques de collecte ethnographique et invitent ainsi à relativiser les oppositions entre collecte ethnographique et acquisition d'œuvres d'art. La correspondance de Denise Paulme, membre de la mission Griaule de 1935, propose plusieurs exemples du rôle de l'émotion esthétique dans le travail de collecte. Le plus fameux concerne la sculpture dogon collectée par Denise Paulme et Deborah Lifchitz à Yayé :

12. Benoît de I'Estoile, " "Une petite armée de travailleurs auxiliaires" : la division du travail et ses enjeux dans l'ethnologie française de l'entre-deux-guerres », Cahiers du centre de recherche historique, $\mathrm{n}^{\circ}$ 36, pp. 31-59. [http://ccrh.revues.org/3037\#quotation]; Vincent Debaene, " "Etudier des états de conscience" : la réinvention du terrain par l'ethnologie, 1925-1939 », L'Homme, n 179, 2006, pp. 7-62.

13. Barbara Kirshenblatt-Gimblet, "Objects of Ethnography", dans Ivan Karp \& Steven D. Lavine (dir.), Exhibiting Cultures. The Poetics and Politics of Museum Display, Washington \& London, Smithsonian Institution Press, 1991, p. 386-443.

14. Marcel Mauss, Manuel d'ethnographie, Paris, Payot \& Rivages, 2002 (1947).

15. Marcel Griaule et Michel Leiris, Instructions sommaires pour les collecteurs d'objets ethnographiques, Paris, Musée d'ethnographie et Mission scientifique Dakar-Djibouti. Voir à ce sujet Jean Bazin, «N'importe quoi », dans Marc-Olivier Gonseth, Jacques Hainard et Roland Kaehr (éd.), Le Musée cannibale, Neuchâtel, Musée d'ethnographie de Neuchâtel, 2002, pp. 273287 , et Jean Jamin, «La règle de la boîte de conserve », L'Homme, n 170, 2004, pp. 7-10. Dans le même ordre d'idées, Paul Rivet écrivait en 1929 que, « pour l'ethnologue, la maison du pauvre est aussi, sinon plus, précieuse à étudier que le palais du riche » ; «'étude des civilisations matérielles : ethnographie, archéologie, préhistoire », Documents, n² 2, 1929, p. 133.

16. IFAN, Conseils aux chercheurs, Dakar, IFAN, 1948, p. 59.

17. M. Griaule, «Cours technique esthétique » du 4 et du 11 mars 1943, archives de la Bibliothèque Éric-de-Dampierre (Nanterre). 
«Nous avons une dizaine de statuettes aux bras levés, presque toutes en très bon état, certaines admirables figureront très honorablement dans la vitrine d'Art africain. Enfin - que Ratton verdisse - nous avons une statue de $1 \mathrm{~m} 30$, de style jusqu'alors inconnu et antérieure sans doute à la venue des Dogon, le corps très allongé et incurvé, les bras collés au corps, hermaphrodite ; la tête dégage une émotion profonde qui fait de cette pièce un chef-d'œuvre, au sens véritable du mot. Elle était enterrée jusqu'au cou derrière une case, et pour l'avoir nous avons creusé le sol avec nos mains; les habitants nous ont permis de la prendre, sans oser y toucher eux-mêmes. » Lettre de Denise Paulme et Deborah Lifchitz à Georges-Henri Rivière, 2 juillet $1935^{18}$.

Des tensions entre la logique sérielle recommandée et la recherche de l'unicité ou de l'originalité s'observent ainsi dans les collectes ethnographiques. À l'inverse, une attention est souvent accordée au contexte et à la documentation dans l'acquisition d'objets d'art, certains marchands ou collectionneurs constituant des fichiers détaillés au sujet de leur collection.

Les écrits normatifs des ethnologues au sujet des pratiques de collecte concernent également le travail de modélisation des fiches de collecte, devant servir de base à la rédaction des fiches d'inventaire une fois les objets rapportés dans les musées occidentaux. Il s'agit d'établir " pour chaque objet et pour chaque série, des fiches descriptives détaillées sur lesquelles seront mentionnés non seulement le lieu, le moment de la collecte, le nom de l'objet et des matériaux utilisés pour sa confection, mais encore son rôle et les représentations qui s'y rattachent ${ }^{19}$ ". Une histoire du fichage des objets mériterait d'être écrite. Elle mettrait en évidence l'émergence et le développement d'un paradigme ethnique dans la conception de la culture matérielle des sociétés africaines, et sa continuation jusqu'à nos jours ${ }^{20}$. Létablissement d'une équivalence entre le style des objets collectés et le groupe ethnique de leurs producteurs ou de leurs utilisateurs, constitutif de l'histoire de l'art africain mais dénoncé depuis les années 1980, reste en effet trop souvent encore en vigueur ${ }^{21}$. Cette histoire du fichage des objets est également une histoire photographique, de nombreux objets collectés étant photographiés en usage avant d'intégrer les caisses de transport, qu'il s'agisse de documenter des gestes techniques ou d'archiver la façon dont un masque est porté. La photographie (ou le dessin, ou le film) est alors susceptible de fonctionner comme un témoignage visuel sur l'objet ou comme une preuve de son authenticité. Les scènes ou les sorties de masque sont parfois reconstituées à la demande des ethnologues pour compléter le travail de documentation, tandis que certains marchands peu scrupuleux transforment de bonnes copies de masque en objets authentiques en photographiant une danse commandée spécialement ${ }^{22}$.

\section{Collectes mixtes: une histoire indisciplinée}

L'histoire des collectes est souvent écrite dans une perspective disciplinaire : les historiens des sciences retracent celle des collectes botaniques ou zoologiques, les historiens d'art s'intéressent à l'acquisition des œuvres d'art, les anthropologues aux collectes ethnographiques. Cette logique disciplinaire a cependant parfois pour conséquence d'occulter le caractère souvent hybride des pratiques de collecte. Non seulement le jugement esthétique des ethnologues peut jouer dans leur choix d'objets à collecter ou le souci documentaire compter pour tel ou tel amateur d'art premier, mais les collectes sont souvent plus largement mixtes. Ainsi, par exemple, durant la période coloniale, les administrateurs coloniaux ou

18. Denise Paulme, Lettres de Sanga, Paris, Fourbis, 1992, p. 85

19. Germaine Dieterlen, « Méthode moderne de I'ethnographie », dans Henri Lauga (dir.), De la banquise à la jungle. Les Français, la terre et les hommes, Paris, Plon, 1952, pp. 168-169.

20. Phillip L. Ravenhill, 1996, "The Passive Object and the Tribal Paradigm. Colonial Museography in French West Africa", M. J. Arnoldi, C. M. Geary \& K. L. Hardin (éd.), African Material Culture. Bloomington and Indianapolis, Indiana University Press, 1996, pp. 265-282.

21. Sidney Littlefield Kasfir, "One Tribe, One Style? Paradigms in the Historiography of African Art", History in Africa, vol. 11, 1984, pp. 163-193 ; Jean-Paul Colleyn, « Images, signes, fétiches. À propos de l'art bamana (Mali) », Cahiers d'études africaines, n 95, 2009, p. 734.

22. Jeremy MacClancy, "A Natural Curiosity. The British Market in Primitive Art", Res, n 15, 1988, p. 171 . 
les missionnaires, puis les ethnologues eux-mêmes, n'ont pas seulement rassemblé des collections d'artefacts : ils ont également collecté ou capturé des animaux sauvages, des plus petites bêtes jusqu'aux plus grands mammifères. Les missions de collecte sont le plus souvent pluridisciplinaires.

Le cas des deux missions scientifiques suisses en Angola est révélateur de la conjonction, sur le terrain, entre ethnographie et zoologie. La première, en 19281929, est avant tout une expédition cynégétique, mais les chasseurs et le zoologiste qui la composent rapportent, outre de nombreux spécimens zoologiques pour le musée d'histoire naturelle de la Chaux-de-Fonds, une belle collection d'objets ethnographiques au musée d'ethnographie de Neuchâtel. Albert Monnard, le directeur du premier musée, organise une deuxième mission en 1932-1933, à laquelle participe le conservateur du second musée, Théodore Delachaux. Les collectes ethnographiques sont cette fois-ci les plus importantes, notamment de belles séries de masques, mais de nombreux spécimens zoologiques ou entomologiques sont également ramenés en Suisse ${ }^{23}$. À la même époque, une logique identique de collecte mixte anime la fameuse mission Dakar-Djibouti, qui constitue un moment important de l'histoire de l'ethnographie française. Si les collectes d'objets ethnographiques sont mises au premier plan, cette mission, comme la plupart des missions ethnographiques organisées en Afrique à la fin de la période coloniale, fournissent également aux ethnologues l'occasion d'obtenir de nombreux spécimens zoologiques ou entomologiques ${ }^{24}$. À l'inverse, les naturalistes profitaient pour leur part de leurs missions sur le terrain pour acquérir des objets ethnographiques. Le professeur Achille Urbain, directeur du parc zoologique de Vincennes et de la ménagerie du Jardin des Plantes, a par exemple rapporté de son expédition de 1937 en Afrique centrale, outre de nombreux spécimens zoologiques vivants ou naturalisés, une dizaine d'objets ethnographiques, en particulier des armes, qu'il a déposés au musée de l'Homme.

Après la Seconde Guerre mondiale, les chercheurs de l'Institut Français d'Afrique Noire sont les premiers à répondre, à des degrés cependant divers, aux exigences de pluridisciplinarité de l'Institut. En 1951, le géographe Jean Dresch écrivait ainsi : "nos connaissances sont trop incomplètes pour que les efforts ne soient pas rassemblés, et qu'à l'exemple de Th. Monod un naturaliste ne dédaigne pas de noter gravures rupestres, légendes et outils, un ethnologue ne renonce pas à ramasser plantes, insectes ou cailloux ${ }^{25}$ ". La mission Dekeyser-Holas dans l'Est libérien, organisée dans le cadre de l'IFAN en 1948, rend bien compte de cette volonté : elle est composée d'un zoologiste et d'un ethnologue et rassemble des collections particulièrement variées. Pendant la période coloniale, collectes ethnographiques et captures zoologiques ou entomologiques ont donc souvent été effectuées ensemble. Une série d'interférences pratiques et cognitives peut alors être observée dans de telles activités de collecte mixte. Un même objet, un autel de chasseur par exemple, peut être vu de différentes façons, et les crânes d'animaux qui le composent être collectés comme spécimens zoologiques ou comme objets ethnographiques. Des outils identiques sont utilisés indifféremment pour les collections ethnologiques ou zoologiques, qu'il s'agisse des contenants servant à ranger et à transporter les objets ou des instruments de mesure, utilisés aussi bien pour relever la taille des spécimens abattus que pour prendre les mensurations de sujets humains, dans le cadre des collectes anthropométriques.

Le vocabulaire utilisé pour désigner les pratiques de collecte rend bien compte de telles interférences ${ }^{26}$. L'emploi du terme trophée témoigne, par exemple, de l'influence du modèle de la chasse sur les pratiques de collecte, alors susceptibles

23. Manuel Laranjeira Rodrigues de Areia et Roland Kaehr, Masques d'Angola, Neuchâtel, Musée d'ethnographie, 2009 ; M.-O. Gonseth, B. Knodel et S. Reubi (dir.), Retour d'Angola, op. cit. note 8 .

24. J. Bondaz, « L'ethnographie comme chasse. Michel Leiris et les animaux de la mission Dakar-Djibouti », Gradhiva, n. S., n 13, 2011, pp. 162-181, et « L'ethnographie parasitée ? Anthropologie et entomologie en Afrique de l'Ouest (1928-1960) », L'Homme, n² 206, 2013, pp. $121-150$

25. Jean Dresch, «Les publications de I'Institut français d'Afrique noire », Annales. Économies, Sociétés, Civilisations, 6 (2), 1951, p. 225.

26. J. Bondaz, «Prélèvement, capture, collecte. Quels mots pour dire la sélection du patrimoine de collection ? », dans Martin Drouin (dir.), La sélection patrimoniale, Québec, éditions Multimondes, 2011, pp. 11-24. 
d'être interprétées comme des activités de prédation ${ }^{27}$. Lors des collectes de masques, la séparation de la tête de masque de son costume peut ainsi être assimilée à une décollation, à la découpe d'un trophée ${ }^{28}$. De même, lorsque les collecteurs se font photographier à côté des objets collectés, on retrouve parfois le modèle du portrait de chasseur, du tableau de chasse ${ }^{29}$. Autre exemple, l'usage du mot spécimen permet également de pointer les rencontres, sur le terrain, entre ethnographie et sciences naturelles. L'idée de spécimen ethnographique témoigne également de l'influence du modèle naturaliste sur les collectes ethnographiques ${ }^{30}$. Les fiches de collecte et d'inventaire, parfois utilisées indifféremment pour des artefacts et pour des produits de la nature, peuvent révéler ces interférences. Une telle idée fait également référence à des pratiques muséales inspirées des musées d'histoire naturelle, autour de la notion de spécimen de civilisation, développée par Paul Rivet et Georges-Henri Rivière au Musée d'ethnographie du Trocadéro. On trouve aussi en usage, jusqu'à aujourd'hui, la notion de spécimen d'art. Il n'est d'ailleurs pas rare que les collectionneurs conjuguent plusieurs pratiques de collection : art premier et art contemporain, statuaire et perles, art africains et collections naturalistes, trophées de chasse, tableaux de papillons... Le cas de Jacques Kerchache, collectionneur d'arts extra-occidentaux mais aussi de papillons, est de ce point de vue symptomatique.

\section{Rapts, razzias ou pillages : la collecte comme activité prédatrice}

Les pratiques de collecte doivent évidemment être replacées dans leur contexte politique. Elles sont souvent révélatrices d'enjeux de pouvoir, notamment à l'époque coloniale, militaires, missionnaires et ethnographes s'accaparant parfois violemment certains objets. Les exemples d'objets collectés sans l'accord de leurs propriétaires ou saisis militairement sont nombreux. Un cas fameux est celui des statues dédiées aux rois d'Abomey et collectées comme un véritable butin de guerre par des officiers français, lors de la conquête du Dahomey ${ }^{31}$. Ces vols ou ces actes de pillage ne sauraient cependant résumer l'ensemble des pratiques de collecte en contexte colonial. Il convient en outre de tenir compte d'autres formes de violence, symboliques cette fois, comme le montre notamment, dans l'histoire des collectes, l'occultation toujours d'actualité du rôle pourtant central des auxiliaires sur le terrain, qu'il s'agisse des collectes ethnographiques ${ }^{32}$ ou zoologiques $^{33}$, et des intermédiaires dans les circuits d'approvisionnement du marché de l'art africain ${ }^{34}$. Une certaine continuité dans le recours à la force ou l'exercice de l'autorité dans les activités de collecte continue par ailleurs d'être observée après les décolonisations. Nous pourrions ainsi avancer que le cas des hommes politiques s'appropriant des objets appartenant aux collections nationales s'inscrit dans une même logique, qu'il s'agisse par exemple d'Houphouët Boigny prélevant des masques ou des statues dans les collections du musée d'Abidjan pour les offrir à des hôtes prestigieux, les pièces étant alors remplacées par des copies, ou des accusations récemment portées contre Abdoulaye Wade, le président sénégalais déchu en 2012. Ces privatisations de biens publics relèvent ainsi de pratiques corruptives qui déplacent la question des modes de collecte vers la problématique de l'inaliénabilité des collections.

27. Brigitte Derlon et Monique Jeudy-Ballini, «Quoi ? - L'éternité », dans Alain Babadzan (textes réunis par), Insularités : hommage à Henri Lavondès, Nanterre, Société d'ethnologie, 2003, p. 109.

28. Boris Wastiau, Medusa en Afrique. La sculpture de l'enchantement, Genève/Milan, Musée d'ethnographie de Genève/5 Continents, 2008, chap. 2.

29. A.-M. Bouttiaux, Persona. Masques d'Afrique : identités cachées et révélées, Tervuren/Milan, Musée royal de I'Afrique centrale/5 Continents, 2009, p. 31-41.

30. B. de I'Estoile, « "Une petite armée de travailleurs auxiliaires"... », art. cité note 12.

31. Maureen Murphy, «Du champ de bataille au musée : les tribulations d'une sculpture fon » dans Thierry Dufrêne et Anne-Christine Taylor (éd.), Cannibalismes disciplinaires. Quand I'histoire de l'art et l'anthropologie se rencontrent, Paris, musée du quai Branly, 2010, pp. 347-359.

32. B. de I'Estoile « "Une petite armée de travailleurs auxiliaires"... », art. cit. note 12.

33. Nancy J. Jacobs, "The Intimate Politics of Ornithology in Colonial Africa", Comparative Studies in Society and History, vol. 48, n 3, 2006, pp. 564-603.

34. Christopher B. Steiner, African Art in Transit, New York, Cambridge University Press, 1994, p. 9. 
Ces pratiques posent plus généralement la question du vol comme mode de collecte, avec pour conséquence principale l'absence de documentation concernant les objets. Paradoxalement, le vol d'objets rituels fonctionne comme une garantie de leur authenticité. Mais dans le contexte ouest africain, les conceptions locales de telles pratiques mettent en avant les risques encourus non seulement par les voleurs, mais aussi par les marchands et les collectionneurs qui se procureraient de telles pièces. Dans la mesure où ces objets sont considérés comme étant encore " chargés ", leur pouvoir est réputé provoquer des maladies, la folie ou la mort de qui s'en empare. Au pays Lobi, au Burkina Faso, une maladie nommée thil pemere, la grossesse du fétiche, est par exemple supposée atteindre les voleurs, qui auraient le ventre qui gonfle, puis mettraient au monde des crapauds ou des margouillats, avant de décéder ${ }^{35}$. Dans toute l'Afrique de l'Ouest, les récits et les rumeurs concernant de tels objets dangereux sont nombreux. Au Mali, un quinquagénaire nous a par exemple rapporté le cas suivant :

"Dans les années 1970, des voleurs avaient pris un masque dogon caché dans une grotte, dans la falaise. Le masque avait été revendu, et d'intermédiaire en intermédiaire, il était arrivé jusque dans la collection d'un Français ou d'un Belge, je ne sais plus. Mais peu de temps après, le masque est revenu. Un jour, il est revenu tout seul dans la grotte. Il avait fait tout le chemin à l'envers. Et tous les vendeurs, et les voleurs, tous sont morts. Le masque les a tués. "

L'idée selon laquelle une certaine intentionnalité peut être attribuée aux objets en fonction de la manière dont ils sont collectés, en l'occurrence en fonction du risque qu'il peut y avoir qu'ils soient encore "chargés ", est d'ailleurs récurrente dans les discours des agents des musées ouest africains ${ }^{36}$. Elle invite à questionner les conceptions locales des pratiques de collecte et les différences que les populations établissent entre elles.

Dans le contexte actuel de la mondialisation et de la marchandisation de l'art africain, les accusations de pillage sont réactualisées. Les marchands d'art africain continuent souvent d'être accusés de piller le continent africain, sans qu'une approche plus nuancée ne soit proposée, ni que l'on s'interroge sur l'existence, sur place, de marchands africains d'art africain. Les discours de ces derniers sont pourtant révélateurs des stratégies de distinction dans ces logiques d'accusation. En 2012, à l'occasion de l'une de nos enquêtes en Afrique de l'Ouest, un antiquaire sénégalais nous a par exemple expliqué considérer que seuls les marchands occidentaux étaient des pilleurs, ce qui était une façon de légitimer sa propre activité. L'un de ses collègues burkinabè distinguait, pour sa part, parmi ses clients européens ceux qui achetaient en gros, qu'il accusait de pillage, et ceux qui n'achetaient qu'un ou deux objets, perçus comme des amateurs, d'autant plus respectables qu'ils s'inscrivaient dans un rapport qualitatif aux objets. Analysées dans leur contexte d'énonciation, les accusations de pillage permettent ainsi de mieux comprendre et de mettre en perspective les rapports de force qui traversent les collectes contemporaines.

\section{Faire son marché : I'économie des collectes}

La problématique marchande invite elle aussi à entrer dans le détail des pratiques de collecte. L'achat d'objets soulève en effet des questions épistémologiques importantes, dans la mesure où il donne à voir des écarts entre le moment de la collecte et celui de l'entrée en collection. Si l'ethnologue qui achète tel ou tel objet sur un marché pour le compte d'un musée peut être défini comme le collecteur de l'objet, qu'en est-il de l'antiquaire français qui s'approvisionne auprès de l'un de ses homologues africains ? Placer le curseur sur le moment de la collecte devient difficile, sans compter qu'il est habituel pour certains antiquaires de présenter

35. Michèle Cros et Bertrand Royer, «La grossesse du fétiche "fait bois" au Burkina », Psychopathologie africaine, XXXIV, 2, 2007-2008, pp. 257-267.

36. J. Bondaz, « Animaux et objets marrons. Résistances à la mise en exposition dans les zoos et les musées d'Afrique de l'Ouest », Civilisations, 61-1, 2012, pp. 121-136. 
comme une collecte l'achat de copies artificiellement vieillies par des artisans talentueux. De la même manière, comment analyser l'entrée en collection d'objets saisis par des douaniers, par exemple lorsqu'ils sont issus de fouilles archéologiques clandestines ? Souvent opposés à l'achat ou au vol, et présentés comme des modalités d'acquisition plus vertueuses, le troc et le don peuvent camoufler des rapports de force, invitant à s'intéresser aux coulisses de ces collectes. L'histoire du troc à l'époque coloniale rend bien compte de l'asymétrie des échanges et des différents registres de valeur entre collecteurs et populations locales. À l'occasion, la rhétorique du don peut pour sa part servir à occulter l'origine obscure de l'objet donné. C'est le cas, au musée national du Mali, du bélier " de Chirac ». Offerte en 1996 à Jacques Chirac par ses collaborateurs et publiée dans Paris Match, cette statue de terre cuite a été identifiée par le professeur Jean Polet comme étant issue de fouilles clandestines à Tenenkou, au nord de Mopti, et a fini par être restituée à l'état malien. Elle se retrouve cependant exposée comme don du Président de la République française.

De plus, s'il convient de distinguer le vol et l'achat dans les modalités d'acquisition des collections, il est cependant nécessaire de décrire les contraintes qui peuvent peser sur leurs propriétaires, les obligeant ainsi à céder un objet auquel ils sont attachés. La mise en place d'un impôt de capitation pendant la période coloniale et les difficultés rencontrées par les populations colonisées pour se procurer l'argent nécessaire expliquent de nombreuses ventes conclues par les collecteurs. « Pour tous les objets, les Noirs demandent de l'argent, car le souci de payer l'impôt est grand ", résumait Albert Monnard à propos de ses collectes en Angola $^{37}$. Autre exemple, le coût important des funérailles dans de nombreuses sociétés ouest africaines provoque encore aujourd'hui la vente de masques ou de statues à des antiquaires par des villageois soucieux d'offrir des funérailles convenables à un parent décédé. Ces pratiques marchandes génèrent néanmoins toutes sortes de ruses et de stratégies, qui permettent d'expliquer certains malentendus sur le terrain, révélateurs des conceptions variées des pratiques de collecte. L'ethnologue Jean-Paul Lebeuf rendait ainsi compte de ses difficultés de collecteur au Tchad, à la fin des années 1940 : " Le bruit courut bien vite que j'achetais des objets, n'importe lesquels, les plus communs comme les plus précieux. Beaucoup vinrent au campement apporter un pagne, un ornement de plumes, une calebasse, une marmite ${ }^{38}$. " Autre exemple, plus récemment, AnneMarie Bouttiaux a témoigné de difficultés identiques à propos d'une mission de collecte menée chez les Guro de Côte d'Ivoire à la fin des années 1990 :

"Parmi ces difficultés, la plus évidente est assurément celle qui me confrontait aux villageois désireux d'honorer mes requêtes et qui venaient me trouver avec leurs vieux ustensiles domestiques hors services : plats en bois brisés, vieilles cuillers, tabourets mal dégrossis, etc. Face à ma mine peu réjouie, ils insistaient, arguant qu'il s'agissait d'objets anciens réalisés sur place et que c'est bien ce que j'avais demandé. Commencer à invoquer la dimension esthétique, la nécessité d'une belle patine et autres critères propres aux historiens de l'art était passablement incongru. J'ai donc régulièrement consenti à acheter ce type de culture matérielle pour ne pas vexer mes hôtes ${ }^{39}$. "

Ces pratiques marchandes de collecte donnent plus largement à voir des techniques spécifiques, qui varient en fonction des catégories de vendeurs, des lieux d'achat - marché, village artisanal, galeries d'art en Afrique ou en Europe - et des stratégies de mise en valeur de la marchandise. Avec l'essor d'Internet, on assiste en outre depuis une dizaine d'années au développement de nouvelles stratégies marchandes et à l'appropriation de sites d'enchères en ligne ou des réseaux sociaux par les antiquaires africains, qui rendent d'autant mieux

37. Cité dans Serge Reubi, « La mécanique du départ», dans Marc-Olivier Gonseth, Bernard Knodel et Serge Reubi, Retour d'Angola, op. cit. note 8, pp. 88.

38. Jean-Paul Lebeuf, Quand I'or était vivant, Paris, Éditions Je sers, 1950, p. 90.

39. A.-M. Bouttiaux, « Récolter les "choses" et laisser les esprits en paix chez les Guro (Côte d'Ivoire) », art. cité note 10 
compte de la dimension transnationale du marché. L'importance du style de l'échange marchand, le jeu du marchandage et les stratégies de communication des vendeurs influent sur la construction de la valeur des objets. En outre, en Afrique de l'Ouest, un hiatus important existe entre les détenteurs des objets et les collectionneurs : de la même manière que la plupart des collectionneurs occidentaux d'art africain ne sont jamais allés en Afrique, leurs homologues africains ne se rendent pas dans les villages. Ce hiatus explique le développement, de longue date, d'un réseau marchand, local et transnational, qui fonctionne comme une chaîne de médiations. Des distinctions existent cependant entre l'art dit traditionnel, pour lequel le collectionneur a recours aux services d'un antiquaire, et l'art contemporain, pour lequel les collectionneurs peuvent directement s'adresser à l'artiste, voire lui passer commande. Pour ce qui concerne l'art africain contemporain, nous assistons ainsi aujourd'hui à une redéfinition des pratiques de collection en termes de mécénat. Dans tous les cas, ces modalités d'acquisition valorisent les relations de clientélisme, qu'il s'agisse d'entretenir une certaine confiance avec des antiquaires réputés ou de fréquenter des artistes renommés ou prometteurs.

Les divers exemples proposés montrent que, loin de constituer une activité univoque, la collecte recouvre une grande variété de pratiques et articule différentes logiques. Écrire l'histoire et faire l'ethnographie de l'entrée des objets en collection permet ainsi de rendre compte des stratégies multiples des collecteurs et de contextualiser leurs pratiques. Les quelques données disponibles pour éclairer le moment de la collecte reproduisent cependant les asymétries mentionnées plus haut, et souvent dénoncées. L'histoire des collectes est en effet toujours écrite du point de vue des collecteurs et tend, le cas échéant, à occulter celui des propriétaires des objets collectés. Certes, les usages qui sont faits des objets sont parfois documentés et leur biographie retracée, mais les émotions et les affects de leur propriétaire manquent à la compréhension fine de l'entrée des objets en collection. Il y a quelque chose qui se perd dans la collecte, l'acquisition ayant souvent pour revers le trouble du détachement ou la violence de l'arrachement.

\section{L'auteur}

Julien Bondaz est docteur en socio-anthropologie de l'Université Lyon 2 et chercheur postdoctorant au sein de l'EA Histoire Culturelle et Sociale de l'Art (Université Paris 1 Panthéon Sorbonne) et de l'Institut Interdisciplinaire d'Anthropologie du Contemporain (EHESS-CNRS), dans le cadre du Labex Création, Arts et Patrimoines. Il mène des enquêtes ethnographiques en anthropologie de l'art et du patrimoine au Mali, au Burkina Faso et au Sénégal, ainsi que des recherches sur l'histoire des collectes ethnographiques. Il a co-dirigé plusieurs ouvrages collectifs, dont le dernier, avec Laurick Zerbini, Afrique en résonance. Collection du musée africain de Lyon, vient de paraitre aux éditions 5 Continents. 